Article

\title{
An Intelligent Sensor for the Ultra-High-Frequency Partial Discharge Online Monitoring of Power Transformers
}

\author{
Jian $\mathrm{Li}^{1}$, Xudong $\mathrm{Li}^{1}$, Lin $\mathrm{Du}^{1}{ }^{1} *$, Min Cao ${ }^{2}$ and Guochao Qian ${ }^{2}$ \\ 1 State Key Laboratory of Power Transmission Equipment and System Security and New Technology, \\ Chongqing University, Chongqing 400044, China; lijian@cqu.edu.cn (J.L.); lixudong@cqu.edu.cn (X.L.) \\ 2 Yunnan Electric Power Research Institute, Kunming 650217, China; cm1961@sohu.com (M.C.); \\ qianguochao@im.yn.csg (G.Q.) \\ * Correspondence: dulin@cqu.edu.cn; Tel.: +86-23-6510-2442
}

Academic Editor: Issouf Fofana

Received: 15 February 2016; Accepted: 11 May 2016; Published: 19 May 2016

\begin{abstract}
Ultra-high-frequency (UHF) partial discharge (PD) online monitoring is an effective way to inspect potential faults and insulation defects in power transformers. The construction of UHF PD online monitoring system is a challenge because of the high-frequency and wide-frequency band of the UHF PD signal. This paper presents a novel, intelligent sensor for UHF PD online monitoring based on a new method, namely a level scanning method. The intelligent sensor can directly acquire the statistical characteristic quantities and is characterized by low cost, few data to output and transmit, Ethernet functionality, and small size for easy installation. The prototype of an intelligent sensor was made. Actual UHF PD experiments with three typical artificial defect models of power transformers were carried out in a laboratory, and the waveform recording method and intelligent sensor proposed were simultaneously used for UHF PD measurement for comparison. The results show that the proposed intelligent sensor is qualified for the UHF PD online monitoring of power transformers. Additionally, three methods to improve the performance of intelligent sensors were proposed according to the principle of the level scanning method.
\end{abstract}

Keywords: ultra-high-frequency (UHF); partial discharge (PD); online monitoring; intelligent sensor; level scanning method; field programmable gate array (FPGA); high-speed voltage comparator

\section{Introduction}

Power transformers are key equipment in power systems. Faults and insulation defects may happen under electrical, thermal, and mechanical stress with the increase in operation time. Partial discharge (PD) online monitoring is an effective way to identify potential faults and inspect insulation defects in power transformers [1-8]. PD is generally a transient phenomenon accompanied by physical and chemical phenomena, such as electrical pulse, electromagnetic wave, ultrasonic signal, mechanical vibration, and light and gas components, which can be detected by corresponding sensors [9-16]. Among those methods, the ultra-high-frequency (UHF) PD detection method becomes an international, advanced research hotspot because of its advantages such as high sensitivity, strong anti-interference ability, high reliability, and ability to recognize and locate discharge sources [17-22].

The frequency of UHF PD detection ranges from $300 \mathrm{MHz}$ to $3 \mathrm{GHz}$ [23]. The acquisition of UHF PD signal is a challenge because of its high frequency and wide frequency band. The waveform recording method is the simplest method to acquire UHF PD signal, but the sampling rate of acquisition device must be several $\mathrm{GHz}$ according to the sampling theorem, which is beyond an ordinary data acquisition card's ability. There are several high-performance high-speed data acquisition cards and digital oscilloscopes satisfying the requirement, but they are not suitable for the online 
continuous monitoring of the UHF PD signal and are mostly used in laboratory research because of such disadvantages as high cost, large amounts of data to store and transmit, and complex data post-processing $[24,25]$. In order to construct the UHF PD online monitoring system, a peak value recording method was presented in [26] in which the peak-value-detect-and-hold circuit was designed to maintain the maximum magnitude of the UHF PD signal in each phase interval for further data processing, but the low sampling rate and error of the peak-valve-detect-and-hold circuit will cause a large loss of information in the UHF PD signal, which leads to less accurate detection results. Moreover, there are other methods proposed to acquire the UHF PD signal: In [27], increasing a frequency mixing circuit in the signal preprocessing module to decrease the frequency of the UHF PD signal to that of an ordinary data acquisition card was proposed, but the error of signal magnitude is large in this method; in [28] a method using the UHF envelope detection circuit and high-speed data acquisition system to acquire UHF PD signal was demonstrated, but it had the same disadvantages as the waveform recording method. There are also several commercial products available for UHF PD online monitoring, but taking into consideration their high price, the mass data transmission, and specialized transport protocol, those commercial products are not cost-effective and not suitable for a wide application of constructing a UHF PD online monitoring system in power transformers.

The present paper proposes a novel, intelligent senor for continuous online UHF PD monitoring of power transformers in substations. It is characterized by low cost, little data for output and transmission, and Ethernet functionality, which is suitable for wide use. Section 2 proposes a new method, namely the level scanning method, to acquire the statistical characteristic quantities of the UHF PD signal, which is the base of the intelligent sensor. Section 3 introduces the implementation of the intelligent sensor. Section 4 presents the comparative experiment between intelligent sensors and the waveform recording method, and the results are presented and discussed. Section 5 presents a further analysis of the experiment results and proposes three methods to improve the performance of the intelligent sensor. The use of the proposed intelligent sensor for UHF PD detection in other electrical components is also discussed in Section 5. The last section offers conclusions.

\section{The Level Scanning Method}

The PD activity has statistical characteristic quantities. The most fundamental and important statistical characteristic quantities are the magnitude of discharge, the number of discharges, and the corresponding phase distribution, from which we can obtain $\phi-V_{\max }, \phi-n_{\mathrm{tot}}, \phi-V-n$, and other pattern charts, where $\phi$ is phase, $V$ is the magnitude of PD signal, $V_{\max }$ is the maximum magnitude of the PD signal in a phase interval, $n$ is number of discharges, and $n_{\text {tot }}$ is the total number of discharges in a phase interval. Some statistical characteristic quantities can be extracted from these patterns charts to recognize the PD pattern or to monitor the evolution of insulation detects long-term to avoid insulation failure. A novel method, namely the level scanning method, is proposed in this paper to obtain the magnitude of discharge, the number of discharges, and the corresponding phase distribution directly without the post-processing of data.

Figure 1 shows the basic principle of the level scanning method. In this figure, the distribution of discharges within a power frequency cycle is shown. The UHF PD pulses appear in both the positive and negative half cycles of the AC voltage. Note that the UHF PD pulses are actually waves with positive parts and negative parts because of decaying oscillation, and the positive pulses here are used to represent actual UHF PD pulses for simplicity in Figure 1. In order to obtain the magnitude of discharge, a set of increasing levels, $V_{1}-V_{5}$, namely the reference levels, are compared with the UHF PD pulses. Initially, the power frequency cycle is divided into several equal phase intervals. Then, the UHF PD pulses, whose magnitudes are larger than a set reference level within several power frequency cycles, are counted and stored for each phase interval according to the time UHF PD pulses appeared on. In this way, the corresponding number of discharges for different reference levels is obtained. Moreover, it can be observed that the number of discharges counted becomes smaller when reference levels increase. If the step of reference level is small enough, the magnitude and number of discharges 
can be precisely extracted. It should be emphasized that, in this method, the number of discharges for a specific magnitude of discharge in a phase interval represents the number of all discharge pulses whose magnitude are greater than the corresponding reference level in this phase interval, and the corresponding reference level is regarded as the magnitude of discharge in this method, which is different from the traditional method.

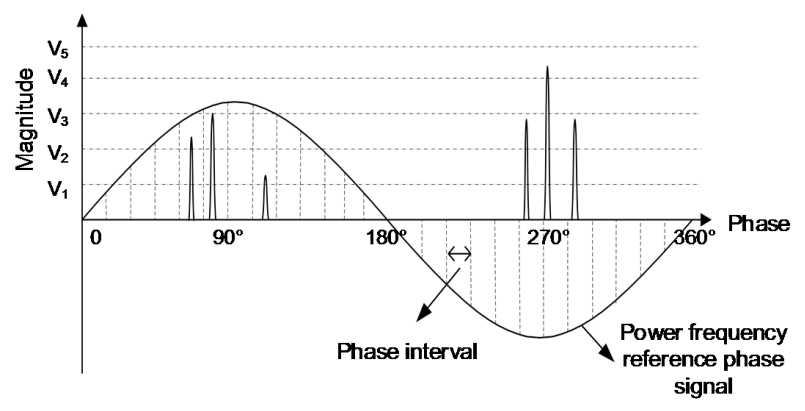

Figure 1. The principle of the level scanning method.

In order to implement this method, the configuration of the designed intelligent sensor is shown in Figure 2. The dotted lines signify analog signals, and the solid lines signify digital signals. Four antennas are used to receive the UHF PD signal and connect to the data processing module via cables. Note that the UHF PD measurement for a power transformer only uses one antenna in our design. The advantage of the four-antenna design is that the intelligent sensor can measure UHF PD for four power transformers at most, which can lower the cost for the construction of the UHF PD online monitoring system for power transformers in substations. The UHF PD signals from the selected channel are input into an ultra-fast voltage comparator (UFVC) after being filtered and amplified for comparison with an analog reference signal output by a digital analog converter (DAC). The input signal of DAC, namely the reference level, is controlled by a field programmable gate array (FPGA). After the comparison, if the magnitude of the UHF PD signal is greater than the reference level, the UFVC will output a square signal that will be scanned by the FPGA, and then the number of discharges for this reference level in corresponding phase interval will add 1 in FPGA's memory. This process continues until the scanning cycles reach the set value, and this process goes on with the next reference level, which is changed by the algorithm in FPGA. The whole process continues until there is no more UHF PD signal that is larger than the reference level.

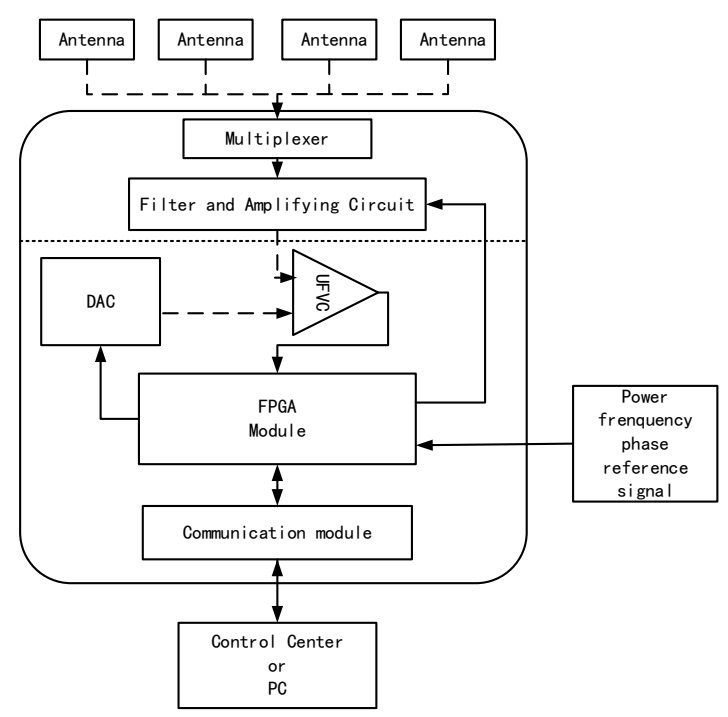

Figure 2. The configuration of the intelligent sensor. 


\section{Implementation of the Intelligent Sensor}

\subsection{Ultra-High-Frequency Antanas}

The UHF antenna is one of the core parts of the proposed intelligent sensor. There are several kinds of antennas designed for UHF PD detection in [29-32]. In this work, the optimal third order Peano fractal antenna designed in our previous work [33] is used as the UHF antenna of the intelligent sensor for its good properties and small size $-90 \mathrm{~mm} \times 90 \mathrm{~mm}$. The voltage standing wave ratio (VSWR) curve of the antenna is shown in Figure 3a, which shows that the antenna has a wide pass band where VSWR $<5$ between $0.3 \mathrm{GHz}$ and $1 \mathrm{GHz}$. The three-dimensional radiation patterns at $0.4 \mathrm{GHz}$ and $0.7 \mathrm{GHz}$ are shown in Figure 3c,d, respectively. The patterns at the two frequencies are both near a hemisphere, which shows that the antenna has a good direction. The prototype of the antenna is shown in Figure $3 b$.

For the installation of this antenna, it is not installed in a transformer drain valve. An alternative way to install PD antennas in power transformers was presented in [32], and the installation of antenna in the proposed intelligent sensor also uses this method. Specifically, a hole will be opened at the wall of the transformer, and a dielectric window made of polytetrafluoroethylene (PTFE) will be welded to install the antenna container. There is a shell of the antenna container made of cast iron that can prevent outside noises from coming into the antenna system.

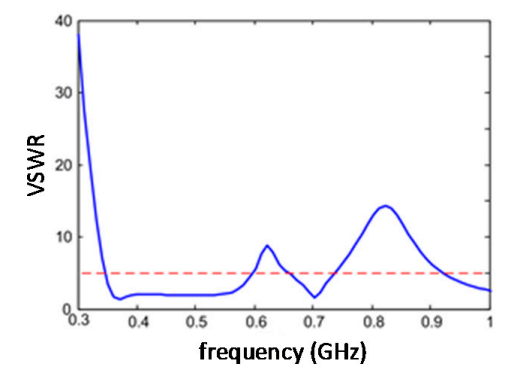

(a)

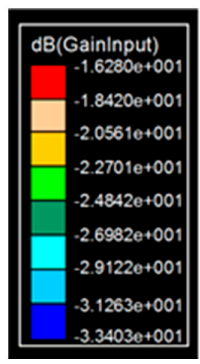

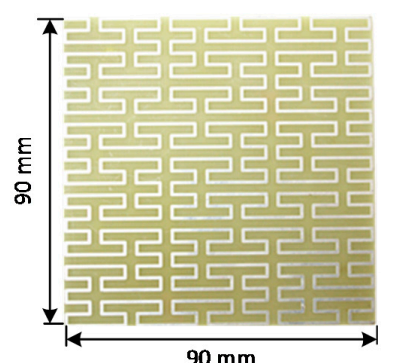

(b)
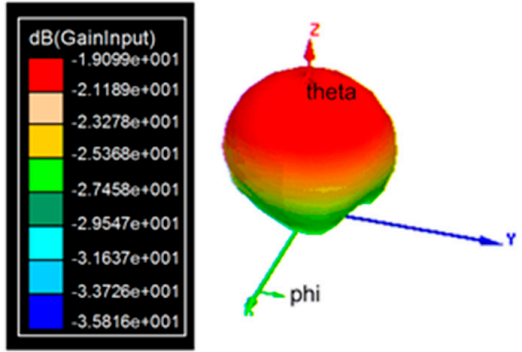

(d)

Figure 3. The third Peano fractal antenna: (a) Voltage standing wave ratio (VSWR) curve of the third Peano fractal antenna; (b) the prototype of the third Peano fractal antenna; (c) the 3-D radiation patterns of the third Peano fractal antenna at $370 \mathrm{MHz}$; and (d) the 3-D radiation patterns of the third Peano fractal antenna at $700 \mathrm{MHz}$.

\subsection{Hardware of the Data Processing Module}

The data processing module is another core part of the proposed intelligent sensor. In our work, the data processing module is divided into two parts, namely the signal pre-processing module and the data acquisition module.

\subsubsection{The Signal Pre-Processing Module}

The signal pre-processing module comprised of a multiplexer, a band-pass filter, and an amplifier circuit is integrated in a printed circuit board (PCB). The analog multiplexer CD452 by Fairchild 
(Semiconductor Corporation, Sunnyvale, CA, USA) is used as a 4-channel multiplexer to choose the signal channel. Considering the interference such as the corona discharge (with the upper frequency below $300 \mathrm{MHz}$ ) and the signals of cell phones (880-960 MHz) [29], the band-pass filter is designed with a pass band from 300-800 MHz. The amplifier circuit composed of two CLC425s (National Semiconductor Corporation, Santa Clara, CA, USA) has an alternative gain according to signal intensity, namely $20 \mathrm{~dB}$ and $40 \mathrm{~dB}$. The operation of the multiplexer and the amplifier is controlled by FPGA.

\subsubsection{The Data Acquisition Module}

The data acquisition module is also integrated in a PCB. As shown in Figure 2, the level scanning process is accomplished by FPGA, DAC, and UFVC. In our design, FPGA controls all of the functions of the intelligent sensor, which contains the control of the signal pre-processing module, the level scanning process, and the communication module. In particular, an Altera FPGA, model EP2S30 of the Stratix II device family, is utilized, which is characterized by a mass equivalent logic element up to $180 \mathrm{k}, 9 \mathrm{Mbits}$ of on-chip, supporting various Input/Output standards along with support for 1-gigabit per second (Gbps) source synchronous signaling with DPA circuitry. Moreover, an UFVC ADCMP567 (Analog Devices, Inc., Norwood, MA, USA) is used, which is characterized by a 250-ps propagation delay input to output, 50-ps propagation delay dispersion, and a 5-GHz equivalent input rise time bandwidth, which is enough to compare the signal with the frequency range from $300 \mathrm{MHz}$ to $3 \mathrm{GHz}$.

\subsection{Software of the Data Processing Module}

The program in the data processing module controls all of the functions of the intelligent sensor, which contains UHF PD signal reception, pre-processing, comparison and scanning, data storage, and data transmission, as shown in Figure 4. The specific steps are as follows:

Step 1 Initialization. In this step, the signal channel, the gain of amplifier, the initial reference level, and the number of scanning cycles are set. Moreover, a frequency cycle is divided into several equal phase intervals, and the corresponding memory addresses to store the number of discharges within each phase interval are assigned in FPGA.

Step 2 Signal comparison, data storage, and transmission. The start of the module is first triggered by the zero crossing point in the rising edge of the power frequency phase inference signal after being initialized, and the scanning time is counted from this moment. UHF PD signals are compared with the reference level and then scanned by FPGA, and the phase of UHF PD signals are calculated simultaneously. If the UHF PD signal is greater than the reference level, the number of discharges within the corresponding phase interval in the memory address add 1 . If the counted scanning cycle reaches the set valve $t_{\mathrm{s}}$, stop scanning and transmitting the data to the PC or control center. The data is a row of numbers, which is shown in Table 1.

Step 3 Comparison with variable reference level. FPGA automatically changes the value of the reference level according to the algorithm. Repeat Step 2.

Step 4 Stop operation. Judge the number of discharges in each phase interval; if they are totally equal to zero, which means there is no discharge whose magnitude is greater than the reference level, stop scanning and exit the loop.

The final statistical parameters of PD collected by the intelligent sensor are reserved as a table in the PC or control center, as shown in Table 1 , where $V_{i}$ is the reference level, $j$ is the serial number of phase interval, and $n_{i j}$ is the number of discharges whose magnitude is greater than $V_{i}$ within phase interval $j$. From this table, we can plot the pattern charts of $\phi-V_{\max }, \phi-n_{\text {tot }}, \phi-V-n$, and other exact statistical characteristic quantities for PD pattern recognition and fault diagnosis. 


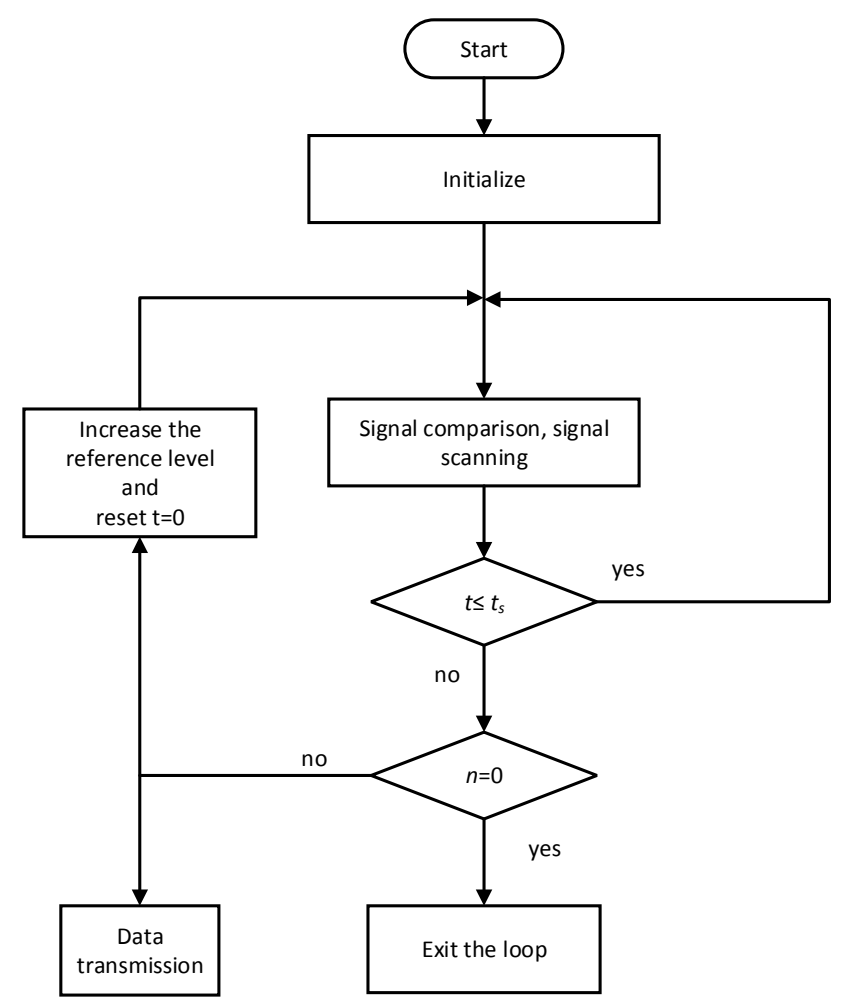

Figure 4. Flowchart of the data processing program.

Table 1. The table of data output from the intelligent sensor.

\begin{tabular}{ccccc}
\hline \multirow{2}{*}{ Reference Level } & \multicolumn{4}{c}{ Phase Interval } \\
\cline { 2 - 5 } & $\mathbf{1}$ & $\mathbf{2}$ & $\ldots$ & $j$ \\
\hline$V_{1}$ & $n_{11}$ & $n_{12}$ & $\ldots$ & $n_{1 j}$ \\
$V_{2}$ & $n_{21}$ & $n_{22}$ & $\ldots$ & $n_{2 j}$ \\
$V_{3}$ & $n_{31}$ & $n_{32}$ & $\ldots$ & $n_{3 j}$ \\
$\ldots$ & $\ldots$ & $\ldots$ & $\ldots$ & $\ldots$ \\
$V_{i-1}$ & $n_{(i-1) 1}$ & $n_{(i-1) 2}$ & $\ldots$ & $n_{(i-1) j}$ \\
$V_{\mathrm{i}}$ & $n_{i 1}$ & $n_{i 2}$ & $\ldots$ & $n_{i j}$ \\
\hline
\end{tabular}

\section{Experiment and Results}

\subsection{Experiment}

The case study is used to assess the performance of the proposed intelligent sensor for UHF PD detection of the power transformer. For comparison, the waveform recording method of UHF PD signal is also used at the same time.

Figure 5 shows the UHF PD detection experiment setup. The experiments are carried out in an electromagnetic shielded laboratory at room temperature $\left(20^{\circ} \mathrm{C}\right)$. The PD free power source consists of a function generator and a high voltage amplifier (TREK $50 \mathrm{kV} / 12 \mathrm{~mA}$, TREK, Inc., Lockport, NY, USA). The function generator generates the AC voltage, and the high voltage amplifier raises the AC voltage to the required level. A PC is connected to the intelligent sensor to store the PD data and perform other data processing tasks. Moreover, the UHF PD signal output from the signal pre-processing module is directly input into oscilloscope (DPO 7104, Tektronix Inc., Beaverton, OR, USA) to record the UHF PD signal. The record length of the oscilloscope reaches up to the 400-M point, and its real-time sampling rate reach up to $10 \mathrm{GS} / \mathrm{s}$ in 4 channels, which is sufficient to collect the UHF PD signal for a power frequency cycle $(20 \mathrm{~ms})$. 


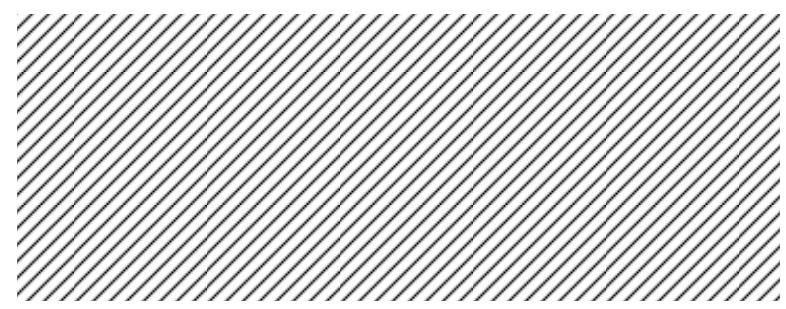

Figure 5. The experiment setup.

There were three typical artificial defect models built in the experiment to generate UHF PD signals. Figure 6a shows an air-void discharge model, including three layers of oil-impregnated paper and a sphere-to-board electrode system. A disc-shaped void with a diameter of $20 \mathrm{~mm}$ was bounded with oil-impregnated paper in the air-void discharge model. Figure $6 \mathrm{~b}$ shows an experiment model of a cylinder-to-board electrode for surface discharge defects. Figure $6 \mathrm{c}$ shows the corona discharge model, which is basically a needle-plate electrode system. The thickness of the pressboard in each model is $0.5 \mathrm{~mm}$. When performing the experiments, the artificial defect models were placed into a test chamber that was made out of organic glass and was filled with insulation oil. The UHF antenna was placed beside the test chamber.

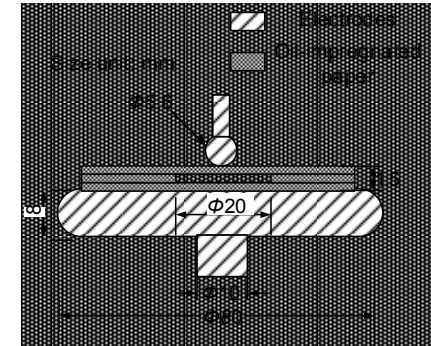

(a)

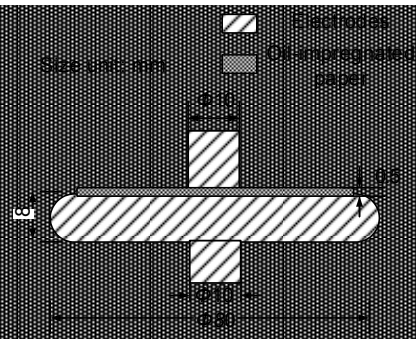

(b)

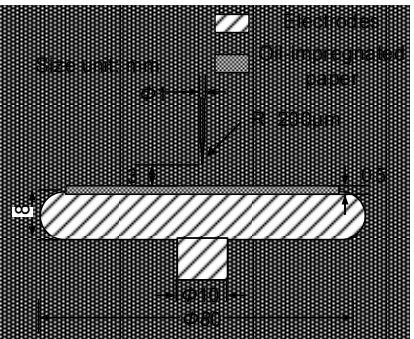

(c)

Figure 6. Three types of artificial defect models in insulation oil: (a) the air-void discharge model; (b) the surface discharge model; and (c) the corona discharge model.

Table 2 shows the inception voltages, breakdown voltages, and test voltages of the three types of artificial defect models in the experiment. During the experiment, the amplification factor of the amplifier is set at $40 \mathrm{~dB}$, and the power frequency cycle is divided into 128 equal phase intervals. The UHF PD signals of 200 power frequency cycles are scanned under each reference level for the surface discharge model and the air-void discharge model. The UHF PD signals of 400 power frequency cycles are scanned under each reference level for the corona discharge model because its discharge frequency is small compared to the other two discharge models. The applied voltage for each discharge model increases gradually from $0 \mathrm{kV}$. When the applied voltage for each discharge model reaches the corresponding test voltage shown in Table 2, stop increasing the applied voltage. When the corresponding test voltage is applied to each discharge model for $15 \mathrm{~min}$, start the intelligent sensor and oscilloscope to collect the UHF PD signals at the same time. Moreover, the magnitude of the background interference signal, which is distributed in the whole cycle, is about $20 \mathrm{mV}$. In order to avoid the background interference signal, the initial reference level is set at $20 \mathrm{mV}$, and the step of reference level is set at $10 \mathrm{mV}$.

Table 2. Partial discharge (PD) experiment conditions.

\begin{tabular}{cccc}
\hline Defect Model & Incepetion Voltage (kV) & Breakdown Voltage (kV) & Test Voltage (kV) \\
\hline Air-void discharge & 6.1 & 12.3 & 9.7 \\
Surface discharge & 8.4 & 13.2 & 10.5 \\
Corona discharge & 5.7 & 12.5 & 9.0 \\
\hline
\end{tabular}




\subsection{Results}

The acquired data of the intelligent sensor are integrated in a data table similar to Table 1. The $\phi-n_{\text {tot }} \phi-V_{\text {max }}$, and $\phi-V-n$ pattern charts of the three types of discharge models are plotted by the drawing program according to the corresponding data tables. For comparison, the UHF PD signals acquired by the oscilloscope for the three types of discharge models are also processed by the level scanning method. The $\phi-n_{\text {tot }}, \phi-V_{\max }$, and $\phi-V-n$ pattern charts of the two methods for the air-void discharge model, the surface discharge model, and the corona discharge model are shown in Figures 7-9 respectively. Because the initial reference level is set at $20 \mathrm{mV}$, the signals whose magnitude are below $20 \mathrm{mV}$ have been reset to 0 in all $\phi-V_{\max }$ and $\phi-V$ - $n$ pattern charts.

From Figures 7-9 it can be seen that the data acquired by the intelligent sensor can strongly reflect the corresponding characteristics of the three types of discharge models as well as the data acquired by the oscilloscope, which validates the effectiveness of the proposed intelligent sensor. The discharge of the air-void discharge model under the given test voltage mainly distributed in the rising and falling edges of the positive and negative half cycles, respectively $\left(0^{\circ}-90^{\circ}\right.$ and $\left.180^{\circ}-270^{\circ}\right)$, and the PD phase distributions in the positive and negative half cycles were basically symmetrical. The discharge of the surface discharge model under the given test voltage mainly distributed in the range of $30^{\circ}-130^{\circ}$ in the positive half cycle and $180^{\circ}-290^{\circ}$ in the negative half cycle. The discharge of the corona discharge model under the given test voltage both concentrated on the peaks in the positive half cycle and the negative half cycle, and the magnitude and number of discharges in the negative half cycle were greater than those in the positive half cycle. However, there are differences in the magnitude and number of discharges between the two methods. The magnitudes of discharges for the three types of discharge models collected by the oscilloscope are all greater than those collected by the intelligent sensor. The number of discharges collected by the intelligent sensor are all much greater than those collected by the oscilloscope, especially for the number of discharges with low magnitude. These differences will be discussed in the next section.

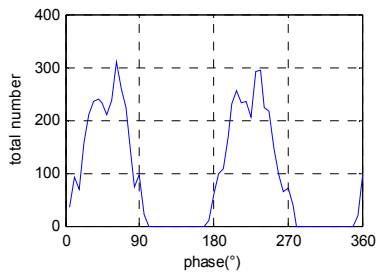

(a1)

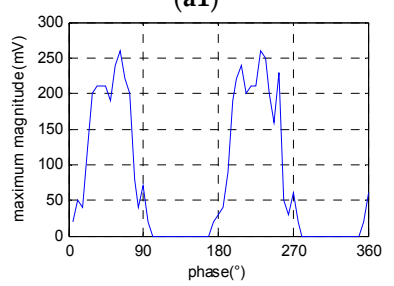

(b1)

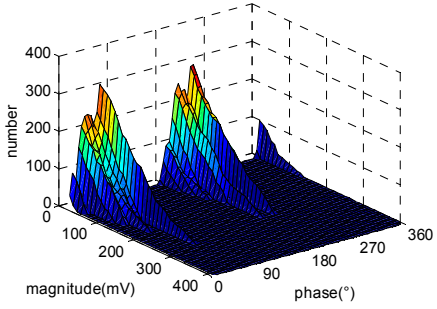

(c1)

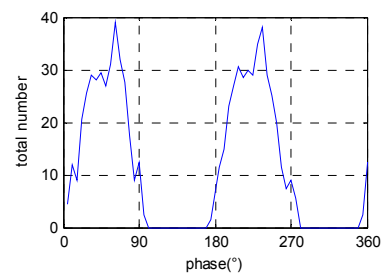

(a2)

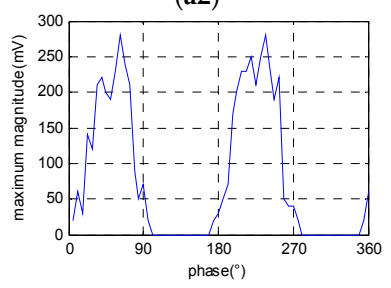

(b2)

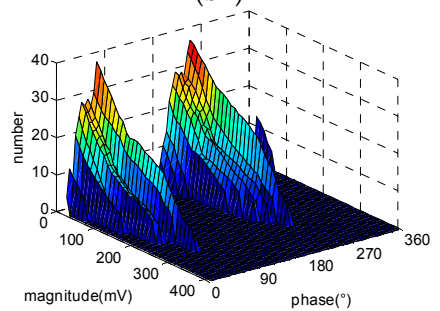

(c2)

Figure 7. PD pattern charts of the air-void discharge model. (a1)-(c1) are the $\phi-n_{\mathrm{tot}}, \phi-V_{\max }$, and $\phi-V-n$ pattern charts of data acquired by the intelligent sensor, respectively; (a2)-(c2) are the $\phi-n_{\mathrm{tot}}, \phi-V_{\max }$, and $\phi-V-n$ pattern charts of data acquired by the oscilloscope, respectively. 


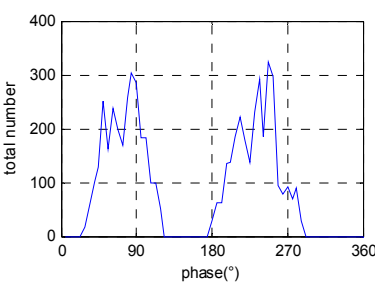

(a1)

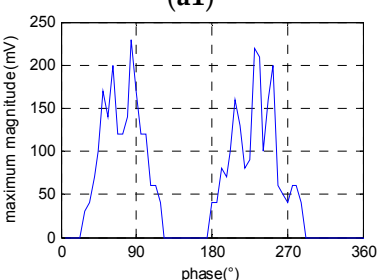

(b1)

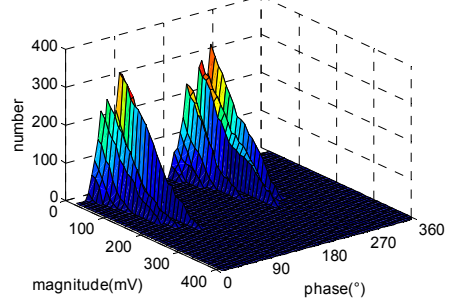

(c1)

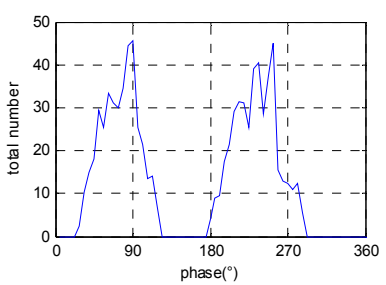

(a2)

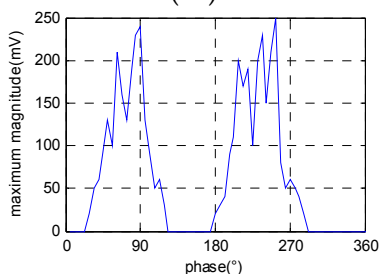

(b2)

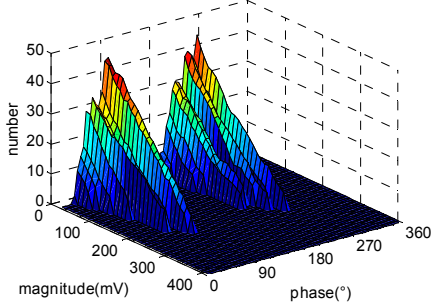

(c2)

Figure 8. PD pattern charts of the surface discharge model. (a1)-(c1) are the $\phi-n_{\text {tot }}, \phi-V_{\max }$, and $\phi-V-n$ pattern charts of data acquired by the intelligent sensor, respectively; (a2)-(c2) are the $\phi-n_{\text {tot }}, \phi-V_{\max }$, and $\phi-V-n$ pattern charts of data acquired by the oscilloscope, respectively.

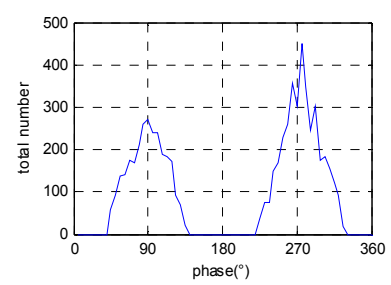

(a1)

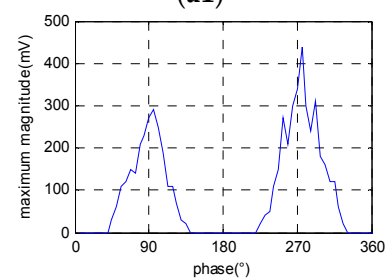

(b1)

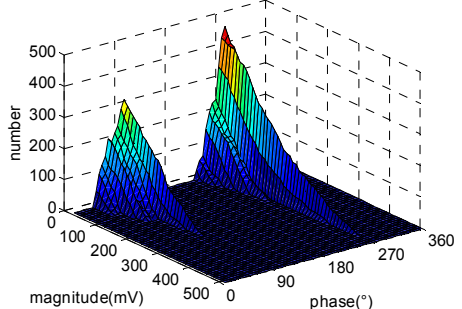

(c1)

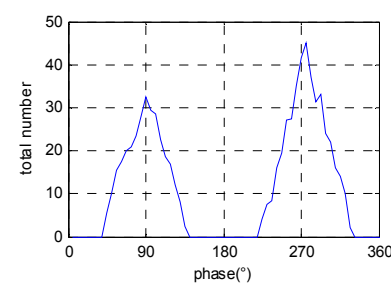

(a2)

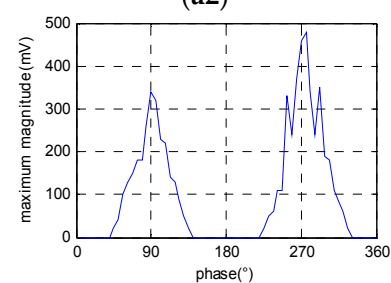

(b2)

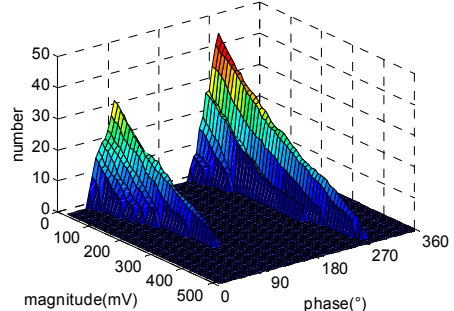

(c2)

Figure 9. PD pattern charts of the corona discharge model. (a1)-(c1) are the $\phi-n_{\text {tot }}, \phi-V_{\max }$, and $\phi-V-n$ pattern charts of data acquired by the intelligent sensor, respectively; (a2)-(c2) are the $\phi-n_{\mathrm{tot}}, \phi-V_{\max }$, and $\phi-V-n$ pattern charts of data acquired by the oscilloscope, respectively. 
$V_{\max }$ is an important parameter in PD, which in general can reflect the severity of defects. In order to quantitatively analyze the differences between $V_{\max }$ acquired by the intelligent sensor and that by the oscilloscope, the correlation of the $\phi-V_{\max }$ pattern charts of the two methods of the three types of discharge models were analyzed, respectively. The correlation of the two figures is always expressed as a correlation coefficient, and the greater the absolute value of the correlation coefficient is, the stronger the correlation is. In our work, Pearson's correlation coefficient was chosen, which was proposed by British statistician Pearson. The formula of Pearson's correlation coefficient between variable $X$ and variable $Y$ is as follows:

$$
\rho_{X, Y}=\frac{\sum X Y-\frac{\sum X \sum Y}{N}}{\sqrt{\left(\sum X^{2}-\frac{\left(\sum X\right)^{2}}{N}\right)\left(\sum Y^{2}-\frac{\left(\sum Y\right)^{2}}{N}\right)}}
$$

The correlation coefficients of the $\phi-V_{\max }$ pattern charts of the two methods of the air-void discharge model, the surface discharge model and the corona discharge model calculated by the above formula are $0.9025,0.8913$, and 0.9127 , respectively, which all show strong correlation. This shows that the intelligent sensor can accurately acquire the UHF PD signals' magnitude.

\section{Discussion}

\subsection{The Influence Factors of the Number of Discharges}

As mentioned previously, the level scanning method counts the number of discharge pulses instead of the number of discharges. Comparing Figures $7 b 1, b 2,8 b 1, b 2$ and $9 b 1, b 2$, respectively, it can be seen that the number of discharges counted by the intelligent sensor is greater than that by the waveform recording method. In order to analyze the reason, a typical UHF PD signal in insulation oil was measured by the oscilloscope, as shown in Figure 10. It can be seen that a UHF discharge signal contains several discharge pulses because of the decaying oscillation of the discharge signal. Therefore, when using the intelligent sensor to count the number of discharges, a UHF PD signal may be counted multiple times. Moreover, the repeated counting times depend on the value of the reference level and the magnitude of the UHF PD signal. For UHF PD signals with same magnitude, the smaller the reference level is, the greater the counted number of discharge pulses is; for a fixed reference level, the greater the magnitude of the UHF PD signal is, the greater the counted number of discharge pulses is.

From the analysis above, it can be seen that, when using the intelligent sensor, the number of discharges with low magnitude is influenced significantly, but the number of discharges with high magnitude is hardly influenced. It is difficult to quantitatively compare the number of discharges by the two methods for their different principles. However, the number of discharges counted by the intelligent sensor can also accurately reflect the characteristics of the PD as the waveform recording method.

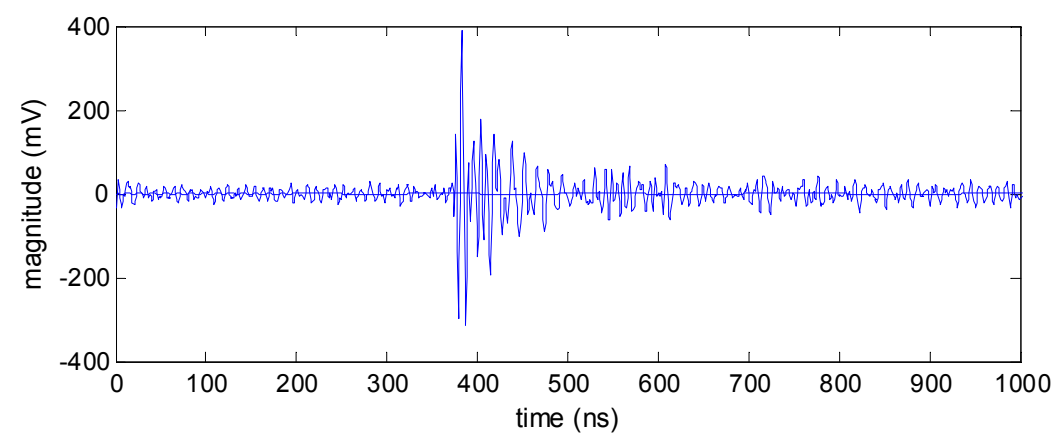

Figure 10. A typical ultra-high-frequency (UHF) PD signal measured by an oscilloscope. 


\subsection{Methods to Improve the Performance of the Intelligent Sensor}

From the experiment result, it is found that the magnitude of discharge acquired by the oscilloscope is greater than that acquired by the intelligent sensor. In order to analyze the reason, the data acquisition theory of the level scanning method is further analyzed.

As shown in Figure 11, $S_{1}, S_{2}, S_{3}$, and $S_{4}$ represent the UHF PD pulse; $V_{1}$ and $V_{2}$ are two reference levels with a different value. When doing a level comparison, the parts of $S_{1}, S_{2}, S_{3}$, and $S_{4}$ that are greater than the reference level are converted into the corresponding square wave in $\mathrm{Sq}_{1}$ and $\mathrm{Sq}_{2}$. If the width of the square wave is greater than the scanning cycle of FPGA, this UHF PD pulse can be counted. It can be seen that, if the width of discharge pulse is narrow or its duration time is short, the width of the corresponding square wave is narrow; for a same discharge pulse, the part that is greater than the reference level becomes narrower when the reference level increases.

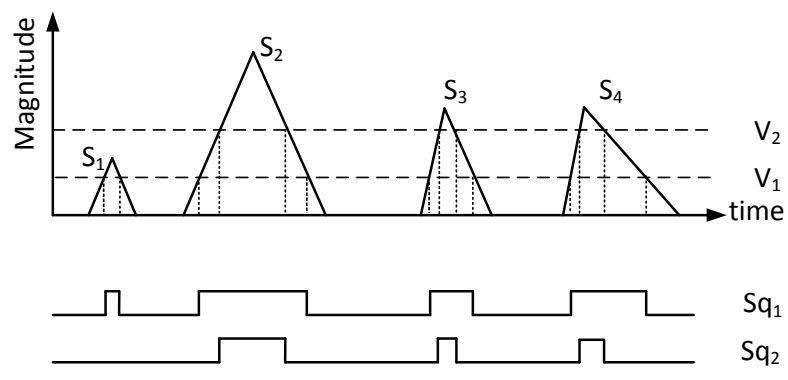

Figure 11. Data acquisition theory of the level scanning method.

In order to quantitatively analyze the performance of the intelligent sensor, we use high-frequency sinusoidal pulses as test signals. A function generator is used to generate high-frequency sinusoidal pulses with different magnitudes and frequencies. Because the core part of the intelligent sensor is the data acquisition module, the high-frequency sinusoidal pulses are directly input into the data acquisition module to obtain the number and the magnitude of the high-frequency sinusoidal pulses.

When doing the test, the function generator is adjusted to output high-frequency sinusoidal pulses whose magnitudes are 250, 500 and $1000 \mathrm{mV}$ and whose frequencies are 50,80 and $100 \mathrm{MHz}$, respectively. Table 3 includes the results obtained by the data acquisition module, where $V_{\mathrm{t}}$ is the theoretical magnitude of the sine wave, $f$ is the frequency of the sine wave, $N_{1}$ is the detection number of the sine waves and $N_{2}$ is the theoretical number of the sine waves. In particular, $V_{\text {off }}$ is the maximum reference level where $N_{1}$ nearly equals $N_{2}$, and $W_{\text {off }}$ is the corresponding width of the part of the sine wave that is greater than $V_{\text {off }}$. Therefore, $W_{\text {off }}$ is the smallest width of the square wave that can be counted by the intelligent sensor. The number of the scanning cycle is 10 power frequency cycles, namely $0.2 \mathrm{~s}$. Table 3 shows $W_{\text {off }}$ is about $2-3 \mathrm{~ns}$. Therefore, for a certain reference level, the discharge pulse may not be detected if its width of the part that is greater than the reference level is smaller than $2 \mathrm{~ns}$.

Table 3. Statistics of sinusoidal pulse by the data acquisition module.

\begin{tabular}{cccccc}
\hline $\boldsymbol{V}_{\mathbf{t}}(\mathbf{m V} \mathbf{)}$ & $\boldsymbol{f} \mathbf{( \mathbf { M H z } )}$ & $\boldsymbol{N}_{\mathbf{1}}$ & $\boldsymbol{N}_{\mathbf{2}}$ & $\boldsymbol{V}_{\mathbf{o f f}}(\mathbf{m V} \mathbf{)}$ & $\boldsymbol{W}_{\mathbf{o f f}}(\mathbf{n s})$ \\
\hline 1000 & 100 & 312,500 & 312,508 & 725 & 2.4184 \\
1000 & 80 & 250,000 & 250,007 & 785 & 2.6583 \\
1000 & 50 & 156,250 & 156,255 & 870 & 3.2824 \\
500 & 100 & 312,500 & 312,510 & 390 & 2.0483 \\
500 & 80 & 250,000 & 250,005 & 420 & 2.2819 \\
500 & 50 & 156,250 & 156,253 & 450 & 2.8713 \\
250 & 100 & 312,500 & 312,506 & 190 & 2.2520 \\
250 & 80 & 250,000 & 250,003 & 210 & 2.2819 \\
250 & 50 & 156,250 & 156,253 & 230 & 2.5638 \\
\hline
\end{tabular}


From the analysis above, the width of the discharge pulse, the scanning frequency of the FPGA, and the step of the reference level influence the performance of the intelligent sensor. In order to improve the performance of the intelligent sensor, three methods are proposed:

- Increase the gain of the amplifier. For a UHF PD signal, the width of the discharge pulse becomes larger after being amplified, and the larger the gain of the amplifier is, the greater the width of the discharge pulse is, which makes the discharge pulse easier to detect.

- Increase the scanning frequency of the FPGA. $W_{\text {off }}$ of the proposed intelligent sensor is decided by the scanning frequency used to count the square wave in the FPGA; the smaller the $W_{\text {off }}$ is, the more accurate the data is. This method can be realized with the rapid development of the FPGA in the future.

- Decrease the step of the reference level. According to the theory of the level scanning method, the detection accuracy of the magnitude will increase with the decrease in the step of the reference level, especially when the reference level is near the peak of the discharge pulse.

When using the proposed intelligent sensor to detect PD in power transformers on-site, the initial reference level is usually set at the magnitude of the background interference signal. The installation location of the antenna on the power transformer is fixed, so the magnitude of the UHF PD electromagnetic wave, which is generated far from the antenna, may become small because of the signal attenuation. In order to sensitively detect the PD generated far from the antenna, the amplification factor of the amplifier is recommended to be set at $40 \mathrm{~dB}$. For the step of the reference level, as mentioned above, the smaller the step of the reference level is, the more accurate the detection of the magnitude is. However, the smaller the step of the reference level is, the longer the time to finish a detection is. Therefore, the selection of the step of the reference level depends on the users' demands for accuracy and efficiency.

\subsection{The Use of the Intelligent Sensor for Ultra-High-Frequency Partial Discharge Detection in Other Electrical Components}

The UHF PD detection method is also used to detect PD in gas-insulation substations (GIS's) and cables because of its high sensitivity and strong anti-interference ability. The proposed intelligent sensor can used to detect UHF PD for GIS's. Because the UHF PD electromagnetic wave in GIS's can leak out at the spacers of a GIS, the Peano fractal antenna can be fixed at the spacer of a GIS and used as an external antenna. However, the intelligent sensor only acquires statistical characteristic quantities of the UHF PD signal and does not acquire the parameters of a single UHF PD electromagnetic wave, so the intelligent sensor cannot be used to localize PD in GIS's.

As for cable, in contrast to the cable itself, insulation failures are more likely happen at the cable connectors that have a higher risk of defects and contamination. As for UHF PD online monitoring for cable connectors, the Peano fractal antenna cannot be used as a coupler, and the inductive UHF sensor and the capacitive UHF sensor are usually used [19]. If the antennas in an intelligent sensor are replaced with inductive UHF sensors or capacitive UHF sensors, which are used for the UHF PD detection of cables, the intelligent sensor can be used to detect UHF PD in cable connectors.

\section{Conclusions}

In this paper, a novel, intelligent senor for UHF PD online monitoring of power transformers based on the proposed level scanning method is introduced. The proposed intelligent sensor accurately acquires and directly outputs statistical characteristic quantities of UHF PD signals containing the distribution of magnitude and number of discharges in power frequency phase intervals without data post-processing, which avoids the error caused by signal delays and achieves low storage and fast real-time commutation for its small data quantity. The intelligent sensor is composed of UHF antennas and integrated circuits with universal chips, which is cheaper compared to the traditional method using high-speed data acquisition cards or an oscilloscope and other industrial products. Moreover, 
the intelligent sensor is easy to install because it is of small volume and is easy to maintain for its high reliability. Because of these advantages, the proposed intelligent sensor can be widely used for UHF PD online monitoring of power transformers and can be used to achieve a distributed PD monitoring system in substations. The main results of the work can be summarized as follows:

(1) A new method to directly acquire the statistical characteristic quantities of UHF PD, namely the level scanning method, is proposed.

(2) The corresponding data processing module of the level canning method was designed, and a prototype was made. Combined with the antenna designed in our previous work, the intelligent sensor was made.

(3) Actual UHF PD experiments with three typical artificial defect models of power transformers were carried out to verify the performance of the intelligent sensor in a laboratory, and the waveform recording method and the intelligent sensor proposed were simultaneously used for UHF PD measurement. The results show that the intelligent sensor can accurately acquire statistical characteristic quantities of the UHF PD signal, which indicates the proposed intelligent sensor is qualified for UHF PD online monitoring.

(4) In order to improve the accuracy of the intelligent sensor, three methods to improve the performance of the intelligent sensor are proposed according to the principle of the proposed level scanning method.

For future works, there is still much work to do to construct a complete UHF PD online monitoring system. Because the level scanning method is different from traditional methods, the corresponding filter methods should be researched, as should the methods of pattern recognition and fault diagnosis.

Acknowledgments: The authors acknowledge the National High-Tech Research and Development Program of China (863 Program) (2011AA05A120). We also appreciate the funding provided by the National Natural Science Foundation of China (No. 51321063) and the National "111" Project of the Ministry of Education of China (No. B08036).

Author Contributions: The research presented in this paper was a collaborative effort among all authors. Jian Li, Xudong Li, and Lin Du proposed the methodology of the intelligent sensor, implemented the intelligent sensor, and wrote the paper. Min Cao and Guochao Qian designed and performed the experiment, discussed the results, and critically revised the manuscript.

Conflicts of Interest: The authors declare no conflict of interest.

\section{References}

1. Barbieri, L.; Villa, A.; Malgesini, R. A step forward in the characterization of the partial discharge phenomenon and the degradation of insulating materials through nonlinear analysis of time series. IEEE Trans. Dielectr. Electr. Insul. 2012, 28, 14-21. [CrossRef]

2. Kiiza, R.C.; Niasar, M.G.; Nikjoo, R.; Wang, X.; Edin, H. Change in partial discharge activity as related to degradation level in oil-impregnated paper insulation: Effect of high voltage impulses. IEEE Trans. Dielectr. Electr. Insul. 2014, 21, 1243-1250. [CrossRef]

3. Sellars, A.G.; Farish, O.; Hampton, B.F.; Pritchard, L.S. Using the uhf technique to investigate PD produced by defects in solid insulation. IEEE Trans. Dielectr. Electr. Insul. 1995, 2, 448-459. [CrossRef]

4. Haida, T.; Wakabayashi, S.; Tsuge, R.; Sakakibara, T. Development of partial discharge monitoring technique using a neural network in a gas insulated substation. IEEE Trans. Power Syst. 1997, 12, 1014-1021.

5. Hikita, M.; Okabe, S.; Murase, H.; Okubo, H. Cross-equipment evaluation of partial discharge measurement and diagnosis techniques in electric power apparatus for transmission and distribution. IEEE Trans. Dielectr. Electr. Insul. 2008, 15, 505-518. [CrossRef]

6. Jacob, N.D.; Mcdermid, W.M.; Kordi, B. On-line monitoring of partial discharges in a HVDC station environment. IEEE Trans. Dielectr. Electr. Insul. 2012, 19, 925-935. [CrossRef]

7. Yao, C.G.; Zhou, D.B.; Chen, P.; Xing, L.; Sun, C.X. UHF-based monitoring for equipment PD within the substation and early warning. High Volt. Eng. 2011, 37, 1670-1676. 
8. Jiang, T.; Li, J.; Zheng, Y.; Sun, C. Improved bagging algorithm for pattern recognition in uhf signals of partial discharges. Energies 2011, 4, 1087-1101. [CrossRef]

9. De Kock, N.; Coric, B.; Pietsch, R. UHF PD detection in gas-insulated switchgear-suitability and sensitivity of the UHF method in comparison with the IEC 270 method. IEEE Electr. Insul. Mag. 1996, 6, 20-26. [CrossRef]

10. Wang, M.; Vandermaar, A.J.; Srivastava, K.D. Review of condition assessment of power transformers in service. IEEE Electr. Insul. Mag. 2002, 18, 12-25. [CrossRef]

11. Chen, M.; Chen, J.; Cheng, C. Partial discharge detection by rf coil in $161 \mathrm{kv}$ power transformer. IEEE Trans. Dielectr. Electr. Insul. 2014, 21, 1405-1414. [CrossRef]

12. Zargari, A.; Blackburn, T.R. Application of optical fibre sensor for partial discharge detection in high-voltage power equipment. In Proceedings of the IEEE 1996 Annual Report of the Conference on Electrical Insulation and Dielectric Phenomena, Millbrae, CA, USA, 20-23 October 1996.

13. Sharkawy, R.M.; Abdel-Galil, T.K.; Mangoubi, R.S.; Salama, M.M.; Bartnikas, R. Particle identification in terms of acoustic partial discharge measurements in transformer oils. IEEE Trans. Dielectr. Electr. Insul. 2008, 15, 1649-1656. [CrossRef]

14. Tang, J.; Zhou, J.; Zhang, X.; Liu, F. A transformer partial discharge measurement system based on fluorescent fiber. Energies 2012, 5, 1490-1502. [CrossRef]

15. Chen, W.; Chen, X.; Peng, S.; Li, J. Canonical correlation between partial discharges and gas formation in transformer oil paper insulation. Energies 2012, 5, 1081-1097. [CrossRef]

16. Sima, W.; Jiang, C.; Lewin, P.; Yang, Q.; Yuan, T. Modeling of the partial discharge process in a liquid dielectric: effect of applied voltage, gap distance, and electrode type. Energies 2013, 6, 934-952. [CrossRef]

17. Meijer, S.; Agoris, P.D.; Smit, J.J.; Judd, M.D.; Yang, L. Application of UHF diagnostics to detect PD during power transformer acceptance tests. In Proceedings of the Conference Record of the 2006 IEEE International Symposium on Electrical Insulation, Toronto, ON, Canada, 11-14 June 2006.

18. Cleary, G.P.; Judd, M.D. UHF and current pulse measurements of partial discharge activity in mineral oil. IEE Proc. Sci. Meas. Technol. 2006, 153, 47-54. [CrossRef]

19. Tenbohlen, S.; Denissov, D.; Hoek, S.M.; Markalous, S.M. Partial discharge measurement in the ultra-high frequency (UHF) range. IEEE Trans. Dielectr. Electr. Insul. 2008, 15, 1544-1552. [CrossRef]

20. Yoshida, M.; Kojima, H.; Hayakawa, N.; Endo, F.; Okubo, H. Evaluation of UHF method for partial discharge measurement by simultaneous observation of UHF signal and current pulse waveforms. IEEE Trans. Dielectr. Electr. Insul. 2011, 18, 425-431. [CrossRef]

21. Beltle, M.; Mueller, A.; Tenbohlen, S. Statistical analysis of online ultrahigh-frequency partial-discharge measurement of power transformers. IEEE Trans. Dielectr. Electr. Insul. 2012, 28, 17-22. [CrossRef]

22. Wang, Y.; Wu, J.; Li, Z.; Yin, Y. Research on a practical de-noising method and the characterization of partial discharge UHF signals. IEEE Trans. Dielectr. Electr. Insul. 2014, 21, 2206-2216. [CrossRef]

23. Judd, M.D.; Yang, L.; Hunter, I. Partial discharge monitoring for power transformers using UHF sensors Part 1: Sensors and signal interpretation. IEEE Electr. Insul. Mag. 2005, 21, 5-14. [CrossRef]

24. Zhang, X.X.; Wang, Z.; Tang, J.; Liu, L.; Wei, Y. GIT partial discharge UHF on-line monitoring system. High Volt. Eng. 2010, 36, 1692-1697.

25. Xie, Y.; Tang, J.; Zhang, X. Development of GIT partial discharge UHF online monitoring system. In Proceedings of the 2010 International Conference on High Voltage Engineering and Application (ICHVE), New Orleans, LA, USA, 11-14 October 2010.

26. Capponi, G.; Schifani, R. Measurement of partial discharge in solid dielectrics with a microprocessor-based system. IEEE Trans. Dielectr. Electr. Insul. 1992, 27, 106-113. [CrossRef]

27. Wang, G.; Hao, Y.; Peng, Y. Application of frequency mixing technique to ultra-high-frequency PD detection for transformers. Proc. CSEE 2013, 24, 115-120.

28. Lixue, L.; Letian, T.; Chengjun, H. Envelope analysis and defects identification of partial discharge UHF signals in GIS. High Volt. Eng. 2009, 35, 260-265.

29. Ye, H.; Qian, Y.; Dong, Y.; Sheng, G.; Jiang, X. Development of multi-band ultra-high-frequency sensor for partial discharge monitoring based on the meandering technique. IET Sci. Meas. Technol. 2014, 8, 327-335.

30. Shibuya, Y.; Matsumoto, S.; Tanaka, M.; Muto, H.; Kaneda, Y. Electromagnetic waves from partial discharges and their detection using patch antenna. IEEE Trans. Dielectr. Electr. Insul. 2010, 17, 862-871. [CrossRef]

31. Li, T.; Rong, M.; Zheng, C.; Wang, X. Development simulation and experiment study on UHF partial discharge sensor in GIS. IEEE Trans. Dielectr. Electr. Insul. 2012, 19, 1421-1430. [CrossRef] 
32. Li, J.; Jiang, T.; Wang, C.; Cheng, C. Optimization of UHF Hilbert antenna for partial discharge detection of transformers. IEEE Trans. Antennas Propag. 2012, 60, 2536-2540.

33. Li, J.; Cheng, C.; Bao, L.; Jiang, T. Resonant frequency calculation and optimal design of peano fractal antenna for partial discharge detection. Int. J. Antennas Propag. 2012, 2012. [CrossRef]

(c) 2016 by the authors; licensee MDPI, Basel, Switzerland. This article is an open access article distributed under the terms and conditions of the Creative Commons Attribution (CC-BY) license (http:/ / creativecommons.org/licenses/by/4.0/). 